\title{
Ivermectin as Pre-exposure Prophylaxis for COVID-19 among Healthcare Providers in a Selected Tertiary Hospital in Dhaka - An Observational Study
}

Mohammed Tarek Alam, Rubaiul Murshed, Pauline Francisca Gomes, Zafor Md. Masud, Sadia Saber, Mainul Alam Chaklader, Fatema Khanam, Monower Hossain, Abdul Basit Ibne Momen, Naz Yasmin, Rafa Faaria Alam, Amrin Sultana, and Rishad Choudhury Robin

\section{ABSTRACT}

Introduction: While multiple vaccines are undergoing clinical trial across the globe, we yearn for an FDA approved drug to protect us from the devastating pandemic for the time being. This study aims to determine the effectiveness of Ivermectin when administered as pre-exposure prophylaxis for COVID-19.

Method: An observational study, with 118 healthcare providers who were enrolled purposively, was conducted in a tertiary hospital in Dhaka from May 2020 to August 2020. The subjects were divided into experimental and control groups; and the experimental group received an oral monthly dose of Ivermectin 12mg for 4 months. Both groups were exposed to COVID-19 positive patients admitted in the hospital during the course of study. The symptomatic subjects were evaluated by physical examination, COVID-19 RT-PCR and/or HRCT of chest. Differences between the variables were determined using the Chi-square test and the level of statistical significance was reached when $p<0.05$.

Result: $73.3 \%$ (44 out of 60 ) subjects in control group were positive for COVID-19, whereas only 6.9\% (4 out of 58) of the experimental group were diagnosed with COVID-19 (p-value $<0.05$ ).

Conclusion: Ivermectin, an FDA-approved, safe, cheap and widely available drug, should be subjected to large-scale trials all over the world to ascertain its effectiveness as pre-exposure prophylaxis for COVID-19.

Keywords: COVID-19, Ivermectin, Prophylaxis, Healthcare worker, Bangladesh.

\section{BACKGROUND}

Submerged in the wave of Coronavirus disease 2019 (COVID-19), the world is weeping. Ever since the outbreak was first identified in December 2019 in Wuhan, China the world has not been the same [1]. An ongoing pandemic, the
Published Online: December 15, 2020

ISSN: $2593-8339$

DOI: 10.24018 /ejmed.2020.2.6.599

Mohammed Tarek Alam*

Bangladesh Medical College Hospital, Shomman Foundation, Bangladesh. (e-mail: mtarekalam16@gmail.com) Rubaiul Murshed

Shomman Foundation, Bangladesh. Pauline Francisca Gomes

Shomman Foundation, Bangladesh. Zafor Md. Masud

Bangladesh Medical College Hospital, Shomman Foundation, Bangladesh.

Sadia Saber

Bangladesh Medical College Hospital, Bangladesh.

Mainul Alam Chaklader

Bangladesh Medical College Hospital, Bangladesh.

Fatema Khanam

Bangladesh Medical College Hospital, Bangladesh.

Monower Hossain

Bangladesh Medical College Hospital, Bangladesh.

Abdul Basit Ibne Momen

Bangladesh Medical College Hospital,

Shomman Foundation, Bangladesh.

Naz Yasmin

International Medical College,

Bangladesh.

Rafa Faaria Alam

Bangladesh Medical College Hospital,

Shomman Foundation, Bangladesh.

Amrin Sultana

Bangladesh Medical College Hospital, Bangladesh.

Rishad Choudhury Robin

Shomman Foundation, Bangladesh

*Corresponding Author
COVID-19 has proved to be the most devastating and unmanageable crisis the world has ever encountered. With the virus proudly lurking around in the atmosphere, millions of lives have already been compromised and many more uncountable innocents are still fighting to survive this war against the invisible rival. The call for an authentic treatment protocol against this virus was made long before 
the crisis was declared as a pandemic by World Health Organization (WHO) [2]. However, as of date, no fixed treatment protocol has proved efficacious enough to be declared as the definitive cure for COVID-19. With researchers across the globe striving to find a potent medicine, doctors have resorted to empirical therapy and symptomatic treatment to combat the disease. However, the key to controlling this pandemic lies solely in stopping the spread of the virus. While the search for an effective vaccine is still underway, it is crucial for researchers and doctors to find an alternative source of prevention using drugs that are already widely available.

Among many drugs being tried and tested, Ivermectin has proved its worth as a safe, efficacious, widely available, potent anti-parasitic drug with multifaceted virucidal properties including its newly studied effect against severe acute respiratory syndrome coronavirus 2 (SARS-CoV-2) as described by Caly et al. [3]. Based on this manuscript, we had conducted an observational study from April 2020 to May 2020 on 100 COVID-19 positive patients in Dhaka who were treated with the combination of Ivermectin and Doxycycline in which all the patients tested negative in RTPCR for COVID-19 by the end of the study. This finding, although conducted in a small-scale, proves the capability of Ivermectin in imposing anti-viral effects against the corona virus [4].

The pioneering drug Ivermectin, is a dihydro derivative of Avermectin which originated solely from a single microorganism, Streptomyces avermitilis, isolated at the Kitasato Institute, Tokyo, Japan by Satoshi Ōmura from Japanese soil and since then, has had an astounding impact in improving the lives and welfare of billions of people across the globe, two of such accomplishments being its use in combating the world's most disfiguring, stigmatized diseases- Onchocerciasis and Lymphatic Filariasis [5], [6].

Furthermore, Ivermectin has proved to be a potent prophylactic drug against River blindness, loa and lymphatic filariasis in several countries of Africa where the drug was distributed through a mass donation program. In mid-to-late 1970s, Dr. William Campbell of Merck Research Laboratories suggested the use of Ivermectin (later named Mectizan) for River blindness in humans. In 1987, Merck stepped forward in the mass donation of Ivermectin through the Mectizan Donation Program (MDP) to the povertystricken parts of the world with the goal to help eliminate River blindness [7]. Later on, due to the MDP's groundbreaking success, mass administration of Ivermectin was expanded to fight against Lymphatic Filariasis in African countries and Yemen where it coexisted with River Blindness. More than thirty years later, several countries in Africa today are making significant progress towards eliminating both diseases. Both River blindness and Lymphatic Filariasis are on WHO's list of Neglected Tropical Diseases targeted for elimination globally [8]. The program (MDP) reaches more than 300 million people in the affected areas annually, with more than 3.4 billion treatments donated since 1987. Moreover, treatment with Ivermectin has been shown to significantly decrease Loa microfilaraemia levels in patients by $90 \%$; pre-treatment testing was done to exclude those with high intensity infections in order to avoid adverse effects [9]. Through these effective mass-treatment programs, Ivermectin has played a critical role in prophylaxis and elimination of River blindness, lymphatic filariasis and low-intensity Loa [6], [10].

This broad-spectrum anti-parasitic drug has exhibited potent antiviral effects against several ribonucleic acid (RNA) viruses, such as Zika virus, influenza A virus, Venezuelan equine encephalitis virus, West Nile virus, porcine reproductive and respiratory syndrome virus, Newcastle disease virus, chikungunya virus, human immunodeficiency virus (HIV-1), yellow fever virus, dengue virus, Japanese encephalitis virus, and tick-borne encephalitis virus [11]-[19]. Ivermectin has also exhibited antiviral activity against deoxyribonucleic acid (DNA) viruses, such as the pseudorabies virus, porcine circovirus 2 , parvoviruses and bovine herpesvirus 1 [20]-[23]. Considering its manifold uses, Ivermectin has indeed proved its worth as "a wonder drug" [6].

Ivermectin has a plasma half-life of approximately 16-18 hours with wide tissue distribution, time-length ranging from 4 days up to 12 days due to its high lipid solubility [24]. Overall, the drug has potent anti-viral effects, preventive capabilities, satisfactory tissue half-life and minimum adverse effects. Compelled by this notion, we have come forth with a research that tests the effectiveness of the Food and Drug Administration (FDA) approved drug Ivermectin in combatting the spread of the SARS-CoV-2 as a pre-exposure prophylaxis for COVID-19.

\section{METHODOLOGY}

An observational study has been conducted in Bangladesh Medical College Hospital (BMCH) from May 2020 to August 2020 among healthy individuals working as healthcare providers in COVID isolation unit. $\mathrm{BMCH}$ has been purposively selected as a study site because it was a prominent tertiary hospital of Dhaka which is the capital of Bangladesh.

Total 118 healthy individuals working as healthcare providers in COVID isolation wards in $\mathrm{BMCH}$ were purposively enrolled in the study. The study group consisted of doctors, interns, nurses, and attendants considering the inclusion criteria which were individuals within the age group of 21 years and 60 years and individuals who were not under treatment with any antiviral drugs. Those who were above 60 years of age and below 21 years of age, pregnant women, or lactating mothers, had chronic liver disease and individuals who were symptomatically ill were excluded from the study.

The individuals were distributed into two groups (experimental and control) comprising of 60 subjects in control group and 58 subjects in experimental group. After taking consent of the individuals under study, the experimental group received a prophylactic dose of Ivermectin $12 \mathrm{mg}$ every 4 weeks for 4 months. During this period all subjects from both groups were exposed to COVID-19 reverse transcription polymerase chain reaction 
(RT-PCR) positive patients admitted in BMCH. The health condition of the subjects was evaluated by assessing symptomatic subjects through physical examination, and/or RT-PCR for COVID-19 and/or High-resolution computed tomography (HRCT) of chest throughout the study period of 4 months.

The data were analyzed using SPSS Version 20 for Windows (IBM Corp., Armonk, NY, USA). Differences between categorical variables were assessed for significance using the Chi-square test. Gender, age, designation, smoking habit, presence of comorbidities, exposure to COVID-19 positive family members, were confounders to determine the significant association of variables with the outcome of being healthy or acquiring COVID-19. The difference was considered to be statistically significant were $\mathrm{p}<0.05$.

\section{RESUlT}

Out of the 118 participants' majority were female. The eldest subject was 60 years old and the youngest was 25 years of age with most participants between the ages of 25 to 40 years. The mean age of experimental group and control group was 37.1 years and 36.7 years respectively. As demonstrated in Table 1, the experimental group consisted of a total of 58 participants with $16(27.6 \%)$ males and 42 $(72.4 \%)$ females, while the control group comprised of total 60 participants with $26(43.3 \%)$ males and $34(56.7 \%)$ females. Among the participants there were 18 doctors, 10 interns, 18 nurses and 12 attendants in experimental group and 11 doctors, 8 interns, 22 nurses and 19 attendants in control group. There were 12 smokers and 46 non-smokers in experimental group, and 13 smokers and 47 non-smokers in control group. In the experimental group $20(34.5 \%)$ participants and in control group $18(30 \%)$ participants had comorbidities. History of exposure to COVID-19 positive family members was found in 11 (19\%) participants in experimental group and $7(12.1 \%)$ participants in control group.

TABLE 1: DEMOGRAPHIC PROFILE (N=118)

\begin{tabular}{|c|c|c|c|}
\hline Variables & $\begin{array}{c}\text { Experimental Group } \\
(\mathrm{n}=58)\end{array}$ & $\begin{array}{c}\text { Control Group } \\
(n=60)\end{array}$ & $\mathrm{p}$-value \\
\hline \multicolumn{4}{|c|}{ Gender } \\
\hline Male $(\%)$ & $16(27.6 \%)$ & $26(43.3 \%)$ & \multirow{3}{*}{0.06} \\
\hline Female $(\%)$ & $42(72.4 \%)$ & $34(56.7 \%)$ & \\
\hline Age (Mean) & 37.1 & 36.7 & \\
\hline \multicolumn{4}{|c|}{ Age Group (Years) } \\
\hline $21-30$ & $13(22.4 \%)$ & $21(36.7 \%)$ & \multirow{4}{*}{0.19} \\
\hline $31-40$ & $31(53.4 \%)$ & $21(33.3 \%)$ & \\
\hline $41-50$ & $6(10.3 \%)$ & $10(16.7 \%)$ & \\
\hline $51-60$ & $8(13.8 \%)$ & $8(13.3 \%)$ & \\
\hline \multicolumn{4}{|c|}{ Designation } \\
\hline Doctor & $18(31.0 \%)$ & $11(18.3 \%)$ & \multirow{4}{*}{0.28} \\
\hline Intern & $10(17.2 \%)$ & $8(13.3 \%)$ & \\
\hline Nurse & $18(31.0 \%)$ & $22(36.7 \%)$ & \\
\hline Attendant & $12(20.7 \%)$ & $19(31.7 \%)$ & \\
\hline \multicolumn{4}{|c|}{ Personal Habit } \\
\hline Smoker & $12(20.7 \%)$ & $13(21.7 \%)$ & \multirow{2}{*}{0.54} \\
\hline Non-Smoker & $46(79.3 \%)$ & $47(78.3 \%)$ & \\
\hline \multicolumn{4}{|c|}{ Co-morbidities } \\
\hline Present & $20(34.5 \%)$ & $18(30 \%)$ & \multirow{2}{*}{0.68} \\
\hline Absent & $38(65.5 \%)$ & $42(70 \%)$ & \\
\hline \multicolumn{4}{|c|}{ Exposure to COVID-19 positive family members } \\
\hline Present & $11(19 \%)$ & $7(12.1 \%)$ & \multirow{2}{*}{0.20} \\
\hline Absent & $47(81 \%)$ & $53(88.3 \%)$ & \\
\hline
\end{tabular}

Out of 58, $54(93.1 \%)$ participants in experimental group remained healthy despite being exposed to COVID-19 RTPCR positive patients. The remaining 4 participants $(6.9 \%)$ of the experimental group emerged symptomatic at the end of the study; among them 3 were females (between age group of 21-40 years) and 1 was male (age group 51-60 years). All 4 participants had mild symptoms with low grade fever, dry cough, and weakness. Additionally, 2 of the 4 participants underwent HRCT of chest which showed bilateral pulmonary infiltrates consistent with COVID-19. On the contrary, the control group, who did not receive the monthly prophylactic dose of Ivermectin from the start of the trial period, a staggering 44 out of 60 participants (73.3\%) belonging to the control group emerged symptomatic at different phases of the study period and tested positive for COVID-19 in RT-PCR. Among the 44 symptomatic subjects, majority belonged to the age group 21-30 years and constituted of $84.6 \%$ (22 out of 26) males (Fig. 1-3). Overall, as shown in Table 2, by the end of the study $73.3 \%$ (44 out of 60) participants in control group were positive for COVID-19, whereas only $6.9 \%$ (4 out of 58 ) of the experimental group were diagnosed with COVID19 which was statistically significant ( $\mathrm{p}$-value $<0.05)$.

TABLE 2: NUMBER OF COVID-19 AND HEALTHY CASES IN CONTROL AND EXPERIMENTAL GROUP $(\mathrm{N}=118)$

\begin{tabular}{cccc}
\hline Groups & COVID-19 & Healthy & p-value \\
\hline Control Group & $44(73.3 \%)$ & $16(26.7 \%)$ & $<0.05$ \\
Experimental Group & $4(6.9 \%)$ & $54(93.1 \%)$ & \\
\hline
\end{tabular}

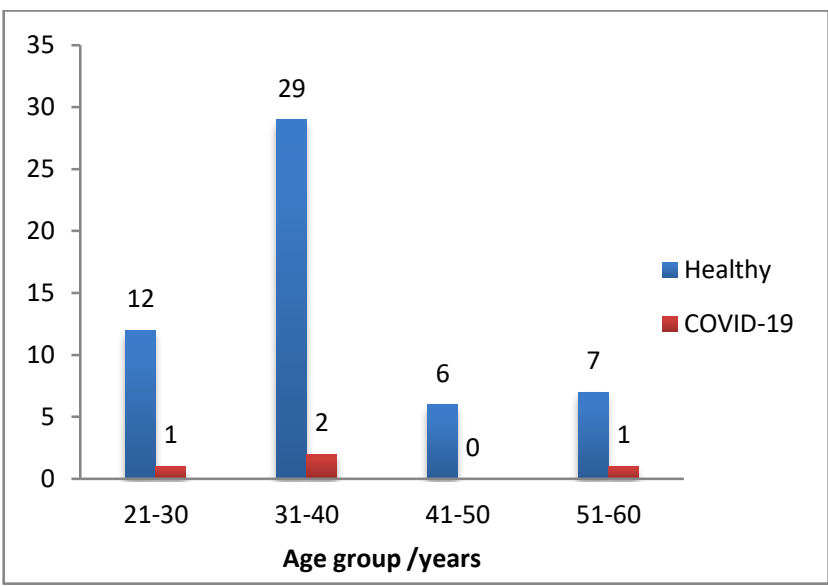

Fig. 1. RT-PCR positive samples among experimental group $(n=58)$.

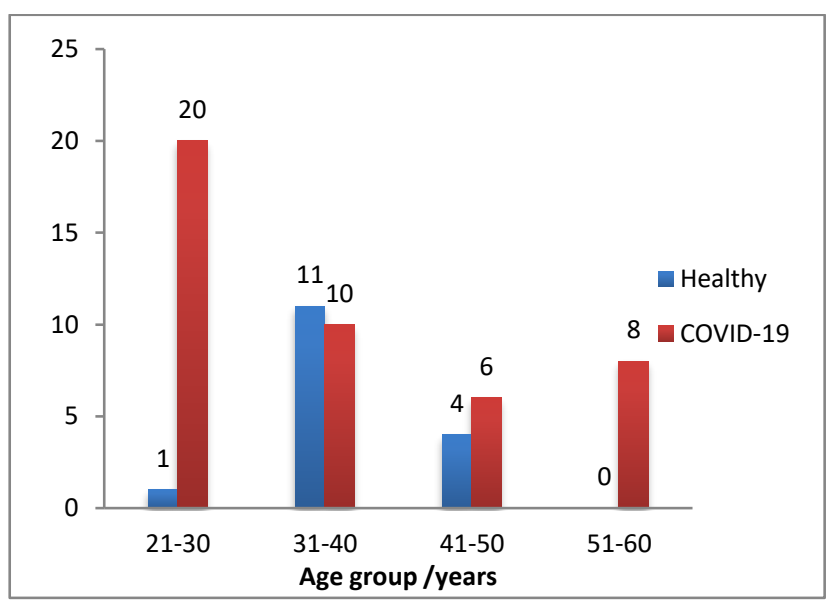

Fig.2. RT-PCR positive samples among control group $(n=60)$. 


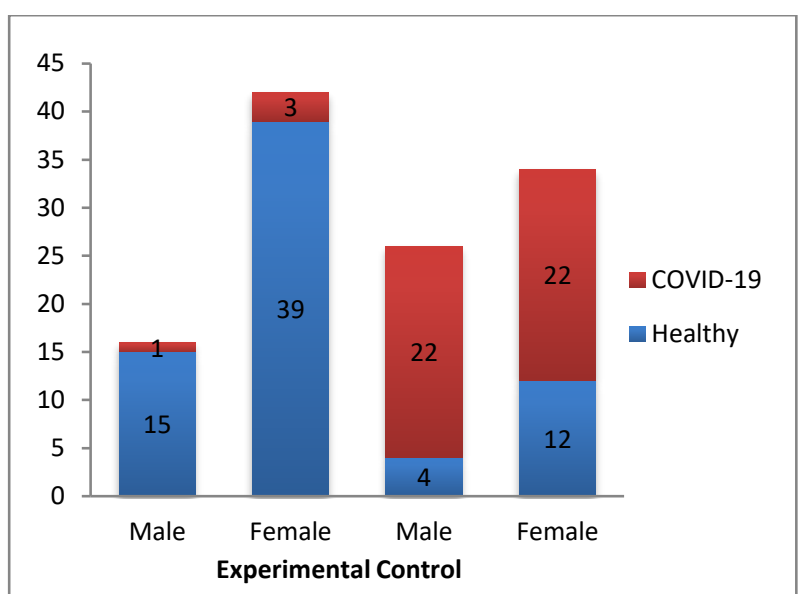

Fig. 3. Frequency of COVID-19 and healthy participants according to gender.

\section{DISCUSSION}

Ivermectin, being a multifaceted medication, has proved to be effective against various organisms [25]. Its safety and efficacy coupled with its low adverse effects make this drug a suitable candidate when orally prescribed. It has rapid oral absorption, high lipid solubility and wide distribution in the body [26]. Ivermectin was identified in late 1970s and first approved for medical use in 1981 [25]. Over the years, thorough studies revealed that Ivermectin possesses broadspectrum endo/ecto-parasiticide activity as well as antiviral, antibacterial, and anticancer effects [3]. Moreover, this drug causes immunomodulation in the host [27].

The causative agent of the current COVID-19 pandemic, SARS-CoV2, is a single stranded positive sense Ribonucleic acid (RNA) virus [28]. In a study, Vero-hSLAM cells were treated with Ivermectin after 2 hours of SARS-CoV-2 infection, resulting in $\sim 5000$-fold reduction in viral RNA after 48 hours. The study revealed that IMP $\alpha / \beta 1$ binds to the coronavirus protein in the cytoplasm and translocate it into the nucleus where the complex disintegrates, thus, freeing the virus off the complex and enabling it to reduce the host cell's antiviral response, leading to enhanced infection [3]. Ivermectin binds to the Imp $\alpha / \beta 1$ heterodimer and destabilizes it, thus preventing Imp $\alpha / \beta 1$ from binding to the viral protein and in the process preventing the virus from entering the nucleus. This leads to decreased infection and increased antiviral response [29].

As the COVID-19 cases surge across the globe, an actual count of the infected still remains unknown due to the alarming characteristic of the virus being spread by individuals despite having mild or no symptoms [30]. This phenomenon has led to the demand for a more collective approach in combating this novel virus by incorporating pharmacological treatment not only for active symptomatic cases but also to prevent and mitigate the virus long before it can initiate the disease process and cause further fatalities. Therefore, as Ivermectin possesses potent anti-viral properties and has also proved to be effective in preventing and eliminating parasitic diseases by mass administration, that too with negligible side effects, we hypothesized its application for the prophylactic approach towards COVID19.
This study has shed a ray of light in portraying Ivermectin's astounding impact on preventing transmission and contraction of COVID-19 in the most vulnerable setting of a hospital among healthcare workers. Moreover, the experimental group did not complain of any side effects or breach of compliance regarding the dosing schedule. However, we acknowledge that this trial has limitations. This study was conducted in a small scale with limited number of subjects being monitored over a short period of time. Standard safety measurements being taken into account, both of the study groups had variegated exposure to COVID-19 positive patients of different severity levels during the trial period. Also, due to the limited availability of testing kits for COVID-19 RT-PCR, only the participants who became symptomatic during the course of the study were subjected to RT-PCR and/or HRCT of chest to confirm the diagnosis. High-risk groups comprising of elders above the age of 60 , critically ill patients, patients with chronic liver disease, pregnant and lactating women were not included in the study. Despite being bound by these limitations, our pragmatic approach to the study has diligently paved its way towards a positive outcome. Safe, cheap, and widely available, Ivermectin, is indeed a drug that might save us all from this sinking ship. It is, therefore, a worthy approach to expand the use of this drug for preexposure prophylaxis of COVID-19.

\section{REFERENCES}

[1] World Health Organization. Coronavirus disease (COVID-19) update. 2020; https://www.who.int/bangladesh/emergencies/coronavirusdisease-(covid-19)-update.

[2] World Health Organization. WHO's COVID-19 response. 2020 https://www.who.int/emergencies/diseases/novel-coronavirus2019/interactive-timeline.

[3] Caly L, Druce JD, Catton MG, Jans DA, Wagstaff KM. The FDAapproved drug ivermectin inhibits the replication of SARS-CoV-2 in vitro. Antiviral Res. 2020; 178:104787.

[4] Alam MT, Murshed R, Bhiuyan E, Saber S, Alam RF, Robin RC Case Series of 100 COVID-19 Positive Patients Treatedwith Combination of Ivermectin and Doxycycline. Journal of Bangladesh College of Physicians and Surgeons. 2020;38(Supplement Issue).

[5] Professor Satoshi Ōmura. 2007; http://www.satoshiomura.info/biography/.

[6] Crump A, Ōmura S. Ivermectin, 'wonder drug' from Japan: the human use perspective. Proceedings of the Japan Academy Series B, Physical and biological sciences. 2011;87(2):13-28

[7] MERCK. Over 30 Years: The Mectizan Donation Program. 2019; https://www.merck.com/stories/mectizan/.

[8] Hopkins A. Treating neglected tropical diseases. Community eye health. 2013;26(82):26-27.

[9] Tielsch JM, Beeche A. Impact of ivermectin on illness and disability associated with onchocerciasis. Tropical Medicine \& International Health. 2004;9(4): A45-A56.

[10] MSD. MECTIZAN Donation Program. n.d https://www.msdresponsibility.com/access-to-health/keyinitiatives/mectizan-donation-program/.

[11] Barrows Nicholas J, Campos Rafael K, Powell ST, et al. A Screen of FDA-Approved Drugs for Inhibitors of Zika Virus Infection. Cell Host \& Microbe. 2016;20(2):259-270.

[12] Götz V, Magar L, Dornfeld D, et al. Influenza A viruses escape from MxA restriction at the expense of efficient nuclear vRNP import. Scientific Reports. 2016;6(1):23138.

[13] Lundberg L, Pinkham C, Baer A, et al. Nuclear import and export inhibitors alter capsid protein distribution in mammalian cells and reduce Venezuelan Equine Encephalitis Virus replication. Antiviral Research. 2013;100(3):662-672. 
[14] Nguyen C, Burton T, Kuklinski W, Gray M, Foy BD. Ivermectin for the Control of West Nile Virus Transmission. New Horizons in Translational Medicine. 2015;2(4):127.

[15] Lee YJ, Lee C. Ivermectin inhibits porcine reproductive and respiratory syndrome virus in cultured porcine alveolar macrophages. Archives of Virology. 2016;161(2):257-268.

[16] Azeem S, Ashraf M, Rasheed MA, Anjum AA, Hameed R. Evaluation of cytotoxicity and antiviral activity of ivermectin against Newcastle disease virus. Pak J Pharm Sci. 2015;28(2):597-602.

[17] Varghese FS, Kaukinen P, Gläsker S, et al. Discovery of berberine, abamectin and ivermectin as antivirals against chikungunya and other alphaviruses. Antiviral Research. 2016; 126: 117-124.

[18] Wagstaff Kylie M, Sivakumaran H, Heaton Steven M, Harrich D, Jans David A. Ivermectin is a specific inhibitor of importin $\alpha / \beta$ mediated nuclear import able to inhibit replication of HIV-1 and dengue virus. Biochemical Journal. 2012;443(3):851-856.

[19] Mastrangelo E, Pezzullo M, De Burghgraeve T, et al. Ivermectin is a potent inhibitor of flavivirus replication specifically targeting NS3 helicase activity: new prospects for an old drug. Journal of Antimicrobial Chemotherapy. 2012;67(8):1884-1894.

[20] Lv C, Liu W, Wang B, et al. Ivermectin inhibits DNA polymerase UL42 of pseudorabies virus entrance into the nucleus and proliferation of the virus in vitro and vivo. Antiviral Research. 2018; 159: 55-62.

[21] Wang X, Lv C, Ji X, Wang B, Qiu L, Yang Z. Ivermectin treatment inhibits the replication of Porcine circovirus 2 (PCV2) in vitro and mitigates the impact of viral infection in piglets. Virus Research. 2019; 263: 80-86.

[22] Nguyen KY, Sakuna K, Kinobe R, Owens L. Ivermectin blocks the nuclear location signal of parvoviruses in crayfish, Cherax quadricarinatus. Aquaculture. 2014;420-421:288-294.

[23] Raza S, Shahin F, Zhai W, et al. Ivermectin Inhibits Bovine Herpesvirus 1 DNA Polymerase Nuclear Import and Interferes with Viral Replication. Microorganisms. 2020;8(3).

[24] Baraka OZ, Mahmoud BM, Marschke CK, Geary TG, Homeida MM, Williams JF. Ivermectin distribution in the plasma and tissues of patients infected with Onchocerca volvulus. Eur J Clin Pharmacol. 1996;50(5):407-410.

[25] Crump A. Ivermectin: enigmatic multifaceted 'wonder' drug continues to surprise and exceed expectations. $J$ Antibiot (Tokyo). 2017;70(5):495-505.

[26] Muñoz J, Ballester MR, Antonijoan RM, et al. Safety and pharmacokinetic profile of fixed-dose ivermectin with an innovative $18 \mathrm{mg}$ tablet in healthy adult volunteers. PLoS neglected tropical diseases. 2018;12(1): e0006020-e0006020.

[27] Ivermectin. In: Aronson JK, ed. Meyler's Side Effects of Drugs (Sixteenth Edition). Oxford: Elsevier; 2016:379-390.

[28] Adedokun KA, Olarinmoye AO, Mustapha JO, Kamorudeen RT. A close look at the biology of SARS-CoV-2, and the potential influence of weather conditions and seasons on COVID-19 case spread. Infectious Diseases of Poverty. 2020;9(1):77.

[29] Sharun K, Dhama K, Patel SK, et al. Ivermectin, a new candidate therapeutic against SARS-CoV-2/COVID-19. Annals of Clinical Microbiology and Antimicrobials. 2020;19(1):23.

[30] World Health Organization. Transmission of SARS-CoV-2: implications for infection prevention precautions. 2020 https://www.who.int/news-room/commentaries/detail/transmissionof-sars-cov-2-implications-for-infection-prevention-precautions. 